Case Report

\title{
Apico-Aortic Conduit for Severe Hypertrophic Cardiomyopathy: Sudden Death despite Conduit Patency 31 Years Postoperatively
}

\author{
Gianantonio Nappi, MD, Giovanni Dialetto, MD, Marisa De Feo, MD, PhD, \\ Raffaela Provenzano, MD, Maurizio Cotrufo, MD, Pasquale Santè, MD, \\ and Alessandro Della Corte, $\mathrm{MD}, \mathrm{PhD}$
}

\begin{abstract}
Although small experiences have been described with the use of apico-aortic valved conduit in the treatment of hypertrophic cardiomyopathy (HCM), the long-term follow-up has never been previously reported.

In a young female patient with symptomatic HCM and a prognostically unfavorable phenotype, apico-aortic conduit was chosen instead of conventional myectomy because severe ventricular hypertrophy involved the whole ventricle, making outflow tract cavity virtually absent in systole. Close clinical and imaging follow-up was postoperatively performed. The patient remained asymptomatic, without cardioactive drug therapy for 30 years, also experiencing 2 successful pregnancies. A striking finding was the perfect patency of the conduit at the last follow-up control (31 years), with computed tomography and echocardiography showing no calcification of the porcine Hancock bioprosthesis inside the graft. Nevertheless, the disease slowly evolved towards the dilative phase and the patient experienced sudden death while scheduled for implantation of defibrillator in waiting list for heart transplant.

The present case could suggest that, in selected cases of HCM not treatable by myectomy, apico-aortic conduit may be an option. The relief of the obstruction can provide even longterm freedom from symptoms, however, late evolution to end-stage cannot be prevented.
\end{abstract}

Keywords: hypertrophic cardiomyopathy, apico-aortic conduit, follow-up

\section{Introduction}

Hypertrophic cardiomyopathy (HCM) is a complex, genetically determined disease, including heterogeneous phenotypes with unpredictable natural history, partly due to the complex reciprocal interactions between etiopathogenetic factors. ${ }^{1,2)}$ Echocardiography is central in HCM

Department of Cardiothoracic and Respiratory Sciences, Second University of Naples, c/o V. Monaldi Hospital, via L Bianchi, Naples, Italy

Received: September 29, 2011; Accepted: February 7, 2012 Corresponding author: Alessandro Della Corte, MD, PhD. Via L Bianchi, V Monadi Hospital, Naples 80131, Italy

Email: aledellacorte@libero.it

(C)2012 The Editorial Committee of Annals of Thoracic and Cardiovascular Surgery. All rights reserved. diagnosis and stratification, respectively quantifying left ventricular free wall thickness and, by Doppler method, subaortic obstruction; however, magnetic resonance imaging can have superior sensitivity in some patients. ${ }^{3)}$

Most patients have favorable natural history, highlighting the importance of risk stratification for treatment choice. Syncopal episodes were proven to be prognostically related to sudden cardiac death (SCD), as well as ventricular tachycardia episodes, family history of SCD or HCM, hypotensive response to exercise test, wall thickness $>30 \mathrm{~mm}$ in young age. ${ }^{1,2)}$ Fewer known risk markers exist for the evolution towards the end-stage.

We report a case of severe HCM in a patient with a high-risk profile, who survived 31 years following implantation of an apico-aortic conduit. 


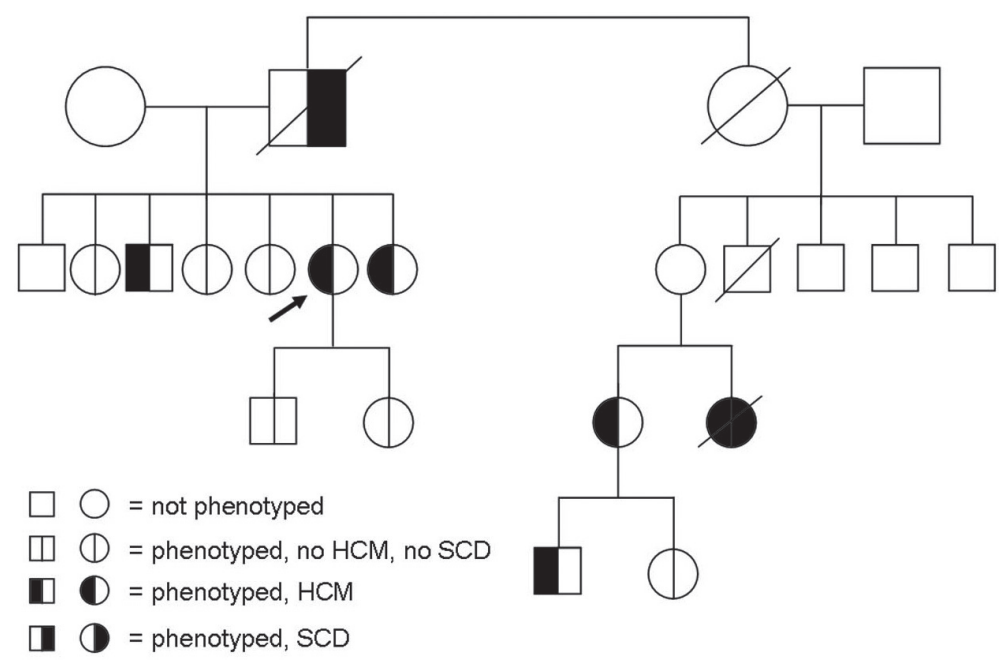

Fig. 1 Partial pedigree. The patient's father died suddenly when he was 52, one sister had received myectomy, one brother was on medical therapy.

\section{Case Report}

The patient was 24-year-old when she was first admitted to our Hospital in October 1979, following a syncopal attack. Three years before, as dyspnea, palpitations, angina and recurrent lipothymic episodes had occurred, propranolol therapy had been started and was at full dose on admission. She had positive familial history for HCM (Fig. 1). Electrocardiography and chest radiography showed signs of left ventricular hypertrophy in sinus rhythm. A haemodynamic study detected obstructive HCM with diffuse muscular thickening and extremely small diastolic ventricular cavity, almost virtual in endsystole, with a peak mid-cavitary gradient of $80 \mathrm{mmHg}$. Our collegial opinion was that a traditional myectomy could not offer satisfactory results, because hypertrophy was circumferential and symmetric, involving both the basal and the mid segments. The patient was informed about this opinion and about the possibility of an unusual treatment modality, previously suggested by Cooley and coworkers ${ }^{4)}$ also for HCM, namely, apico-aortic conduit implantation, and she gave her consent.

The procedure was carried out through a median sternotomy, in extracorporeal circulation and moderate hypothermia, protecting the myocardium by cold antegrade cardioplegia. A 20-mm preclotted, valved Dacron tube with Hancock porcine bioprosthesis (Medtronic Inc.; Irvine, CA) was sutured end-to-side to a longitudinal aortotomy using 4/0 polypropylene running sutures. A circular opening was made at the apex of the left ventricle, where an 18-mm rigid apical connector (Medtronic Inc.; Irvine,
CA) was inserted, with a $35-40^{\circ}$ angle to the septum. The connector was secured with interrupted mattress sutures reinforced with continuous $3 / 0$ braided polyester sutures with Teflon pledgets on the external cardiac surface. Through an opening in the pericardium, the prosthesis was positioned in the pleura, after suturing the proximal and distal conduit segments together, to avoid compression of the pulmonary trunk. Satisfactory haemodynamics were observed, allowing prompt weaning from extracorporeal circulation.

The patient's postoperative course was uneventful, and she was discharged on the 8th postoperative day. The first echocardiographic examination was performed in 1984, and the data collected since then are reported in Fig. 2.

The patient remained asymptomatic, without cardioactive drug therapy for 30 years; in the meanwhile, she experienced 2 pregnancies and delivered 2 healthy babies. On December 2009, however, at the age of 54 years, she presented with atrial fibrillation, first successfully treated by electric cardioversion, then, following a short period of sinus rhythm, recurring to become permanent despite antiarrhythmic therapy. On July 2010, symptoms of heart failure progressively developed. Strikingly, both trans-oesophageal echocardiography and computed tomography (CT) scan showed an unobstructed conduit with no external compression and complete absence of calcifications on the Hancock valve (Fig. 3). The patient entered the waiting list for heart transplant: defibrillator implantation rather than left ventricular assist was chosen as a bridge to transplantation, considering the high arrhythmic risk and the absence of severe or rapidly deteriorating left 

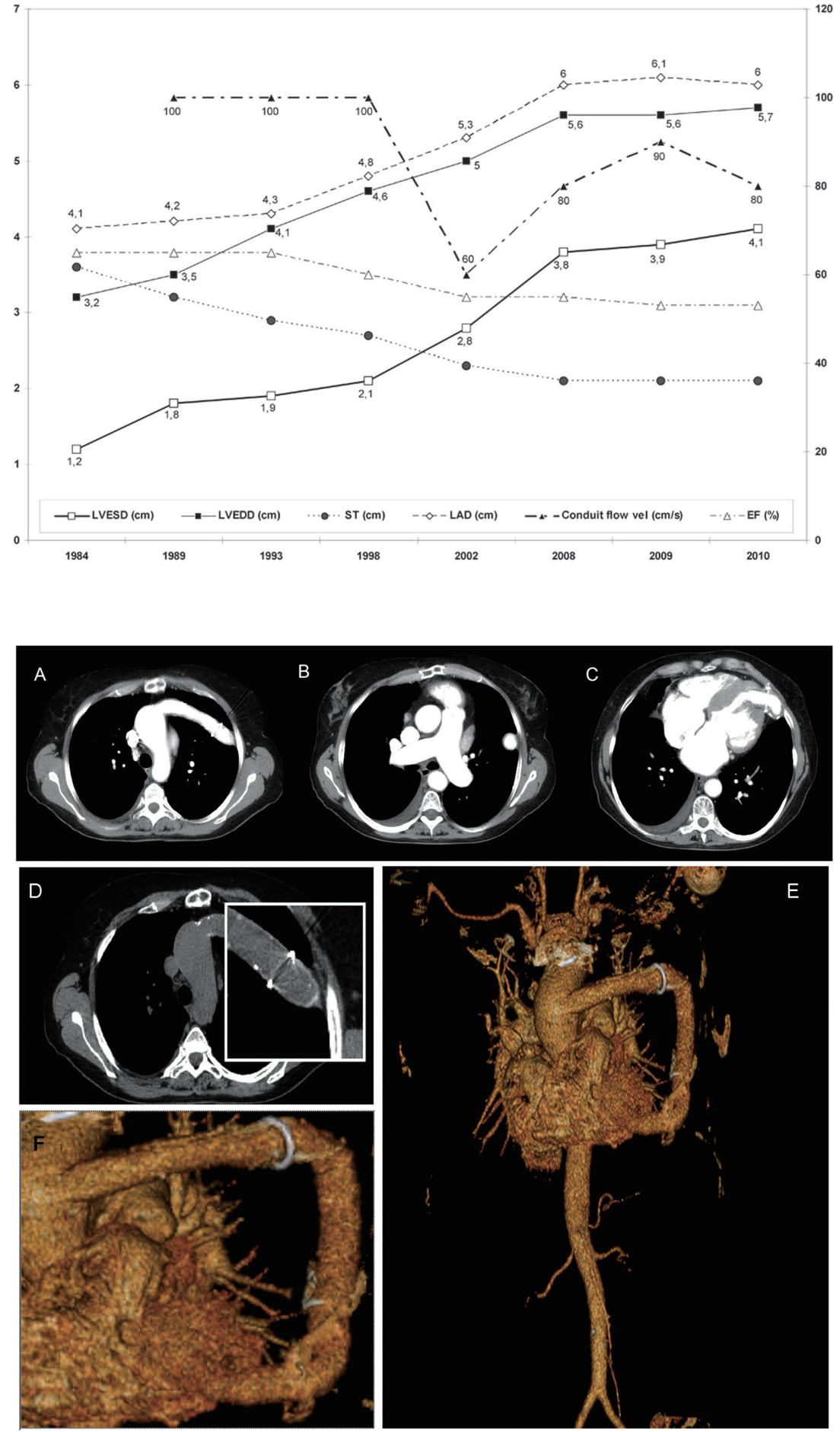

Fig. 2 Echocardiographic postoperative followup. Intra-ventricular gradient was initially $15 \mathrm{mmHg}, 5 \mathrm{mmHg}$ in 1998 and disappeared thereafter. No significant mitral SAM was observed at any timepoint.

Left $y$ axis: LVEDD: left ventricular end-diastolic diameter; LVESD: left ventricular end-systolic diameter; ST: septum thickness; LAD: left atrial diameter

Right $y$ axis: EF :ejection fraction; vel: velocity

Fig. 3 Conduit patency at 31 years of follow-up. Last Angio-CT scan, showing complete contrast enhancement of the entire conduit (A: distal portion; B: middle portion; C: proximal portion). CT-scan without contrast (D) showed absence of bioprosthesis calcification at 34-year follow-up; E shows the 3-dimensional reconstruction and $\mathrm{F}$ the magnified detail of the conduit depicting its patency. ventricular systolic function. Nevertheless, the patient died of SCD the day before the scheduled implantation of a defibrillator. Autopsy was not performed.

\section{Discussion}

Mildly symptomatic HCM forms, with a significant gradient, are generally treated pharmacologically, mainly with calcium channel blockers and beta-blockers. ${ }^{5)}$ For patients at higher risk for SCD, adjunctive therapies include implantable cardiac defibrillator. ${ }^{1)}$ The rarer severe HCM clinical phenotype is usually surgically treated: myotomy-myomectomy is the most widespread technique, indicated for asymmetric forms with predominant septal 
involvement, able to improve gradient and symptoms in about $70 \%$ of cases. ${ }^{2}$

In the above reported case, surgical therapy was indicated because of the clinical severity (positive family history, dyspnea and angina, high SCD risk), after failure of pharmacological therapy. However, apico-aortic conduit was preferred to myectomy because of the unfavorable geometry of hypertrophy and of the previous good short term results obtained by Others. ${ }^{4}$ The implantation of the conduit was chosen as a method to relieve the obstruction and plan a possible subsequent heart transplant when needed.

The operation was immediately followed by clinical improvement, as we had previously acknowledged, when reporting on the short-term results. $\left.{ }^{6}\right)$ Thereafter the patient remained free from symptoms for about 30 years, successfully carrying out two pregnancies. To our knowledge this is the first case ever reported of an apico-aortic conduit implanted for HCM remaining patent and normally functioning for such a long period, with imaging documentation of unobstructed flow through the valved graft. Moreover, the Hancock bioprosthesis showed no sign of calcific degeneration, notwithstanding the two pregnancies, a well-known risk factor for accelerated deterioration.

HCM pathophysiology evolved toward systolic dysfunction and end-stage, although probably more slowly than its natural history would have implied: in fact, basing on the unfavourable prognostic profile a shorter symptom-free survival was expected. The reported case may also suggest consideration of left ventricular assist device implantation for bridge to transplant in patients with this type of history; however, in the specific case, the inherent risks of the redo operation had to be weighed against the moderateness of heart failure. The final adverse outcome in a relatively young age is consistent with the hypothesis of prognosis being independent of outflow obstruction, at least in symptomatic patients. ${ }^{7)}$ Contradictorily, outflow obstruction at rest is a known predictor of both end-stage symptoms and death. ${ }^{8}$ In the reported case, the evolution towards heart failure and SCD, irrespective of hemodynamic factors, may have been genetically determined: a contributing role of the operation (e.g. possible damage from the ventricular incision or the car- dioplegia), is in our opinion, unlikely, given the long time period elapsed between surgery and the end-stage development.

\section{Conclusion}

In conclusion, this case report showed that an apicoaortic conduit, remaining patent at 31 postoperative years, postponed the adverse outcome of HCM, prolonging symptom-free survival, even in a patient with poor natural prognosis.

\section{Disclosure Statement}

The authors warrant that they have no financial or other interest in the manufacture or distribution of the apico-aortic conduit device.

\section{References}

1) Maron BJ. Hypertrophic cardiomyopathy: a systematic review. JAMA 2002; 287: 1308-20.

2) Marian AJ. Hypertrophic cardiomyopathy: from genetics to treatment. Eur J Clin Invest 2010; 40: 360-9.

3) O'Hanlon R, Assomull RG, Prasad SK. Use of cardiovascular magnetic resonance for diagnosis and management in hypertrophic cardiomyopathy. Curr Cardiol Rep 2007; 9: 51-6.

4) Nappi G, Cooley DA. A new surgical technique for treatments of left ventricular outflow tract obstructions. Report of a case and reexamination of our experience. G Ital Cardiol 1977; 7: 1105-12.

5) Marian AJ. Experimental therapies in hypertrophic cardiomyopathy. J Cardiovasc Transl Res 2009; 2: 483-92.

6) Cotrufo M, Nappi G, Scardone M, et al. Intermediate and late follow-up of the use of apico-aortic conduits in the surgical treatment of hypertrophic cardiomyopathy. Int J Cardiol 1986; 12: 35-43.

7) Autore $\mathrm{C}$, Bernabò $\mathrm{P}$, Barillà CS, et al. The prognostic importance of left ventricular outflow obstruction in hypertrophic cardiomyopathy varies in relation to the severity of symptoms. J Am Coll Cardiol 2005; 45: 1076-80.

8) Maron MS, Olivotto I, Betocchi S, et al. Effect of left ventricular outflow tract obstruction on clinical outcome in hypertrophic cardiomyopathy. N Engl J Med 2003; 348: 295-303. 